\title{
Antimicrobial properties of green tea catechins
}

\author{
Peter W. Taylor ${ }^{1}$, Jeremy M.T. Hamilton-Miller ${ }^{2}$, and Paul D. Stapleton ${ }^{1}$ \\ ${ }^{1}$ Microbiology Group School of Pharmacy, 29-39 Brunswick Square, London WC1N 1AX, UK. \\ Tel./Fax 0207753 5867. E-mail peter.taylor@ulsop.ac.uk \\ ${ }^{2}$ Department of Medical Microbiology, Royal Free and University College Medical School, London \\ NW3 2PF, UK.
}

\begin{abstract}
Extracts of leaves from the tea plant Camellia sinensis contain polyphenolic components with activity against a wide spectrum of microbes. Studies conducted over the last 20 years have shown that the green tea polyphenolic catechins, in particular (-)-epigallocatechin gallate (EGCg) and (-)-epicatechin gallate (ECg), can inhibit the growth of a wide range of Gram-positive and Gramnegative bacterial species with moderate potency. Evidence is emerging that these molecules may be useful in the control of common oral infections, such as dental caries and periodontal disease. Sub-inhibitory concentrations of EGCg and ECg can suppress the expression of bacterial virulence factors and can reverse the resistance of the opportunistic pathogen Staphylococcus aureus to $\beta$ lactam antibiotics. For example, relatively low concentrations of ECg can sensitize methicillinresistant $S$. aureus (MRSA) clinical isolates to levels of oxacillin that can be readily achieved in clinical practice. Catechin gallates such as ECg intercalate into phopsholipid bilayers and it is likely that they affect both virulence and antibiotic resistance by perturbing the function of key processes associated with the bacterial cytoplasmic membrane.
\end{abstract}

\section{Keywords}

catechin gallates; Staphylococcus aureus; antibiotic resistance; MRSA; dental caries; periodontal disease; methicillin; bacterial membranes

\section{Origins and nature of tea}

Tea originated in China, possibly as long ago as 2700 BC. Drinking water, boiled for reasons of hygiene, was made more palatable by the addition of leaves from the tea plant. In modern times, tea, in one form or another, is, with the exception of water, the world's most widely consumed beverage; more than two billion cups are drunk daily. For thousands of years, tea has anecdotally been considered to have health-giving properties; this has been amply confirmed in recent years by an accelerating research effort.

The word 'tea' has been used to describe the shrub Camellia sinensis; the fresh leaves of this shrub picked as 'two and a bud' for processing (also termed 'flush'); the processed flush (macerated and heat-dried in the case of green tea); and the beverage made by infusing the processed leaves in boiling water. These multiple definitions may give rise to confusion, especially as there is an additional use of the term 'tea', namely as an aqueous infusion of any herb (thus 'mint tea', 'rooibos tea', etc.) in the sense of the French word tisane. More recently, the discovery of antibacterial activity in the essential oil of the tea tree (Melaleuca alternifolia which is not related to $C$. sinensis) has added further potential for confusion. The material presented in this review is concerned predominantly with work undertaken with aqueous infusions of processed green tea leaves or components thereof. It should be noted that black tea, derived by fermentation of flush before heat drying, contains many of the 
same pharmacologically active components as green tea, but at lower concentrations. Black tea has, in addition, higher molecular weight oxidation products, such as the theaflavins and thearubigens, which are not found in green tea.

\section{Chemistry of green tea}

The chemical composition of green tea is complex and incompletely defined. The most abundant components in green tea are polyphenols, in particular flavonoids such as the catechins, catechin gallates and proanthocyanidins. The fresh leaves contain caffeine (approximately $3.5 \%$ of the total dry weight, or about $50 \mathrm{mg} /$ cup when brewed), theobromine $(0.15-0.2 \%)$, theophylline $(0.02-0.04 \%)$ and other methylxanthines, lignin $(6.5 \%)$, organic acids $(1.5 \%)$, chlorophyll $(0.5 \%)$ and free amino acids $(1-5.5 \%)$, in addition to the unique amino acid theanine (4\%); numerous 'flavour compounds' are also present in much lower amounts (Graham 1992).

Many of the biological properties of green tea have been ascribed to the catechin fraction, which constitutes up to $30 \%$ of the dry leaf weight. These potent antioxidants comprise free catechins such as (+)-catechin, (+)-gallocatechin, (-)-epicatechin (EC) and (-)epigallocatechin (EGC), and the galloyl catechins such as (-)-epicatechin gallate (ECg), (-)epigallocatechin gallate (EGCg), (-)-catechin gallate $(\mathrm{Cg})$ and (-)-gallocatechin gallate (GCg). EGCg is the most abundant of these, comprising about $50 \%$ of the catechin pool; EGC accounts for around 20\%, ECg 13\% and EC 6\% (Hara 2001). Structures of key catechins are shown in Figure 1; note that EGCg differs from ECg only by the presence of an additional hydroxyl group on the aromatic $\mathrm{B}$ ring.

\section{Biological activity of tea components}

\subsection{Therapeutic and biological activities of catechins}

A number of epidemiological surveys have indicated that green tea consumption is linked to lower incidences of various pathological conditions, including cardiovascular disease (Hertog et al. 1993), strokes (Keli et al. 1995), obesity (Bell and Goodrick 2002) and cancer (McKay and Blumberg 2002). Recent clinical studies have revealed physiological responses to tea extracts that may be relevant to the promotion of health, as well as the prevention or treatment of these chronic diseases; furthermore, inconsistencies between some studies should be soon be resolved by improved approaches to their evaluation (McKay and Blumberg 2002). These effects have been attributed, in part, to the antioxidative and free radical scavenging activities of the polyphenolic components (Laughton et al. 1991; Scott et al. 1993). Evidence has recently emerged, however, to suggest that these molecules have the capacity to modulate the physical structure of cell membranes. Thus, a number of membrane-dependent cellular processes, such as cell signalling and the cell cycle (Chung et al. 1999), arachidonic acid metabolism and cell proliferation (Agarwal et al. 1992), and apoptosis and mitochondrial functionality (Spencer et al. 2001) may be influenced by the interaction of catechins with the cellular phospholipid palisade (Caturla et al. 2003).

Within this context, EGCg has been shown to induce apoptotic cell death and cell cycle arrest in tumour cells but not in their normal counterparts; it also favourably affects several signal transduction pathways and is efficacious in animal models of tumour induction (Chen et al. 2004). On the strength of such studies, EGCg has successfully undergone Phase I (human safety) trials (Chow et al. 2003), and patients with asymptomatic Rai stage 0-II chronic lymphocytic leukaemia are currently being recruited to participate in a Phase I/II trial of EGCg to be conducted by the US National Cancer Institute. These activities are creating a precedent for therapeutic interventions with natural polyphenols or their synthetic structural analogues (Williamson and Manach 2005). 


\subsection{In vitro antimicrobial activity of tea extracts}

The antimicrobial activity of tea, suggested for many years by anecdotal evidence, was first demonstrated almost 100 years ago in the laboratory by McNaught (1906), a major in the British Army Medical Corps. He showed that brewed black tea killed Salmonella typhi and Brucella melitensis and recommended that the water bottles of troops be filled with tea in order to prevent outbreaks of infections due to these agents. However, systematic research of the antimicrobial activity of green tea did not begin until the late 1980s; such studies have shown that tea can inhibit and kill a wide range of pathogenic bacteria at or slightly below typical concentrations found in brewed tea (Hamilton-Miller 1997).

The precise antibacterial spectrum of green tea is difficult to assess and there are many conflicting reports of presumptive antimicrobial activity. Some of these anomalies can be explained by differences in definitions of 'susceptible' and 'resistant', and by the various methods of testing that have been employed. However, the present authors' experience is that the activity of tea components is greatly affected by the nature of the test medium, and this is probably the main reason for the variability of results from different centres. It is thus perhaps more helpful to look at results obtained from a single centre (for example, Yam et al. 1997) than those quoted in reviews that are derived from disparate sources. Intuitively, assays based on the use of liquid media that provide an environment for rapid growth of the bacterial strain and maintain the chemical stability of test compounds should yield meaningful data, although no systematic investigation of this issue has so far been undertaken. Notwithstanding these comments, there is unanimity that unfractionated extracts of both green and black tea possess modest antibacterial activity and that staphylococci, including the important nosocomial pathogen, methicillin-resistant Staphylococcus aureus (MRSA), are among the more susceptible bacteria, being inhibited by the equivalent of a 1:10 dilution of a cup of tea. There is good evidence that the catechin components of green tea are responsible for the observed antibacterial activity, and that EGC, EGCg and ECg constitute the most important antibacterial agents (Yam et al. 1997; Hara 2001). The activity of the individual components is, however, also relatively modest. For example, Stapleton et al. (2004a) examined the anti-MRSA properties of a range of naturally occurring and semisynthetic catechins and catechin gallates and found that they possessed either weak activity or no discernable activity; minimum inhibitory concentrations (MICs) ranged from 64 to $>256 \mathrm{mg} / \mathrm{l}$. Such values would preclude these compounds from systemic use as conventional antibacterial agents, but indicate that they may be suitable as topical agents for the treatment of superficial bacterial infections. They may also be able to reduce carriage on body surfaces of opportunistic multi-drug resistant pathogens such as MRSA, where the use of antibiotics could be contra-indicated. In this context, it is interesting to note that the bactericidal action of catechins against MRSA could be markedly improved by substitution of the gallate moiety of ECg with 3-O-acyl chains of various lengths (Stapleton et al. 2004b).

Other bacterial species against which relatively weak direct in vitro antibacterial activity of green tea extracts has been described include Helicobacter pylori (responsible for gastritis and implicated as a risk factor for stomach cancer) and a-haemolytic streptococci (the main aetiological agents of dental caries). Activity against various other microbial pathogens or factors involved in their virulence has been shown; these include viruses such as hepatitis, HIV (Fassina et al. 2002), rotavirus, enterovirus and influenza virus (Song et al. 2005), yeasts (Hirasawa and Takada 2004), filamentous fungi (Okubo et al. 1991), chlamydia, mycoplasmas (Chosa et al. 1992) and parasites (Paveto et al. 2004). The main active principle for these direct antimicrobial effects appears to be EGCg; in black tea extracts, the catechin dimer theaflavin and its gallates are additionally responsible for antibacterial activity.

Food Sci Technol Bull. Author manuscript; available in PMC 2009 October 19. 
These predominantly in vitro data have ramifications for disease prevention in humans. For example, the drinking of green tea appears to protect against dental caries (see below), as well as increasing the number of beneficial bacteria, such as lactobacilli and bifidobacteria, in the gut flora (Goto et al. 1998). The commercially available green tea extracts Sunphenon and Polyphenon E have been formulated as antiseptic creams and mouthwashes and incorporated into facemasks in order to prevent infections; they have also been introduced into vacuum cleaner filters in order to reduce airborne contamination with microorganisms (Hara 2001). Catechin-mediated photo-protection of human skin against bacterial infection has also been claimed (Hsu 2005) and Sharquie et al. (2000) have found that topical tea ointment was as effective for the treatment of impetigo as a topical antibiotic mixture (framycetin and gramicidin) or an oral cephalexin.

\subsection{Anticariogenic activity of green tea}

In the UK and elsewhere, dental caries is one of the most common infectious diseases of man, particularly in deprived urban industrial areas (Downer et al. 2005). It is a multifactorial condition in which diet, nutrition, the resident oral flora and the host response interact to determine whether infection occurs. Any intervention that can reduce its incidence will have a significant impact on public health. Anecdotal reports quoted in the Japanese literature, such as "those who continuously drink a large amount of green tea have less tooth decay" (Kubo et al. 1992) and "drinking green tea makes the mouth clean" (Sakanaka et al. 1989), have spurred research into the potential of tea as an anti-caries agent.

The main bacterial aetiological agents in caries are the a-haemolytic streptococcal species Streptococcus mutans and Streptococcus sobrinus. Lactobacilli and actinomycetes may also be involved. The pathogenesis of the condition involves several sequential steps: bacteria must first adhere to the surface of the tooth and produce a pellicle, a sticky glycocalyx film, composed of a glucan formed by the action of streptococcal glucosyl transferase on dietary sucrose. Such colonization of the surface of the tooth may then lead to the formation of a mixed biofilm that manifests as dental plaque. Within the biofilm, acid is produced by the fermentation of carbohydrates from dietary sources and erosion of the tooth enamel occurs. Streptococci and lactobacilli in the biofilm have the capacity to continue catabolism at low $\mathrm{pH}$ values, so maintaining acid production. It is clear that for pellicle formation and biofilm development, a steady supply of metabolizable carbohydrate is essential; this is provided by the ingestion of sucrose in sugary food and drinks and by the action of salivary and bacterial amylases on dietary starch.

There is convincing evidence that the bioactive components of green tea are able to influence the process of caries formation at several different stages: they may inhibit proliferation of the streptococcal agent, interfere with the process of adhesion to tooth enamel or act as inhibitors of glucosyl transferase and amylase. These observations underpin the results of field trials that have clearly demonstrated the capacity of green tea to reduce the incidence of caries. Several workers have reported that catechins are inhibitory for $S$. mutans and S. sobrinus, with MICs ranging between 50 and $1000 \mu \mathrm{g} / \mathrm{ml}$, well within the concentrations found in brewed tea (Sakanaka et al. 1989; Kawamura and Takeo 1989; Rasheed and Haider 1998). A significant bactericidal effect was found after a brief exposure to $1 \mathrm{mg} / \mathrm{ml}$ of EGCg. Kubo et al. (1992) found that many of the 'flavour compounds' (e.g. nerolidol) found in green tea, although present in too low a concentration to have a direct antibacterial effect, might act synergistically with the abundant catechins.

There is good experimental evidence that catechins prevent the attachment of oral streptococcal pathogens to surfaces. Otake et al. (1991) found that Sunphenon, a commercial mixture of catechins extracted from green tea leaf, prevented the attachment of $S$. mutans to saliva-coated hydroxyapatite discs. The effect was due to modification of the bacterial 
phenotype rather than to the substrate, possibly as a result of catechin-mediated denaturation or deconformation of protein ligands such as surface fimbriae or fibrils. Comparable data was obtained by Xiao et al. (2000), again using $S$. mutans. Similarly, ECg and EGCg adsorbed to protein components of salivary pellicles, modified their physical properties and prevented their further development (Joiner et al. 2004).

Glucosyl transferase is central to caries pathogenesis (Devulapalle and Mooser 2001): in animal models, mutants of $S$. mutans that lack the enzyme are not cariogenic, and such mutants have been proposed for prophylaxis in man as a means to displace wild-type strains. Thus, inhibition of glucosyl transferase represents an attractive anti-cariogenic strategy. Otake et al. (1991) and Hattori et al. (1990) have demonstrated that EGCg and ECg inhibit streptococcal glucosyl transferase, while Kashket et al. (1985) found the catechin fraction of tea and other beverages to be effective inhibitors of the enzyme. Extracts of commercial tea inhibited salivary amylase, according to Kashket and Paolino (1988), although later work (Zhang and Kashket 1998) indicated this might be due to the presence of high molecular weight compounds. However, Hara and Honda (1990) found that monomeric catechins displayed this activity. Zhang and Kashket (1998) have also shown that extracts of various teas inhibit amylase from $S$. mutans.

Experimental in vivo models of caries have been established by the feeding to animals of a cariogenic diet and by infecting specific pathogen-free animals with virulent strains of $S$. mutans. Although these models are somewhat removed from the human situation, significant anticariogenic effects of tea extracts have been found in rats and hamsters (Hamilton-Miller 2001); these observations have encouraged the difficult task of establishing the effects of tea drinking on the development of dental caries in man. Short-term studies in human volunteers have demonstrated an anti-plaque effect of tea extracts. Thus, You (1993) found that using a $0.2 \%$ green tea solution to rinse and brush the teeth reduced the plaque index significantly, while similar findings were made both by Kaneko et al. (1993) with a $0.25 \%$ catechin mouthwash, and Liu and Chi (2000) using tablets consisting of tea polyphenols. Hara and Hattori (1992) obtained a US patent for the use of mixtures containing EGCg, ECg and other catechins as anti-plaque agents. A serious practical disadvantage of using catechins for this purpose is the unpleasant taste of such solutions, a factor that is likely to result in poor patient compliance. Larger, long-term studies are best undertaken in children where caries rates are higher, although this may raise ethical problems. In addition, long-term follow up will be compromised by attrition bias, while there are many confounding factors, both dietary and social, that add to the difficulty of attempting clinical evaluation of agents with potential anticariogenic properties. Credit must therefore be given to those groups who have attempted such studies. Elvin-Lewis and Steelman (1986) reported significantly lower caries and plaque scores in 106 American children who drank tea (1-3 cups/day) than in those who did not. In studies involving almost 800 Japanese children, Onisi et al. (1981) compared dental health of children drinking one cup of 'bancha' tea daily with those who did not consume tea. There was a significantly lower incidence of pits and fissures in the teeth of the juvenile tea drinkers compared to the control group. It should be noted that Onisi (1985) assumed this difference to be due to increased fluoride intake, but does not present any evidence for this. A more recent study from the UK (Jones et al. 1999), investigated the use of tea, and of sugared and carbonated drinks, on dental health in over 6000 children; they clearly established that the drinking of tea was associated with lower levels of caries. The latter study involved black tea, which contains the same monomeric catechins as green tea, but at lower concentrations. Taken together, these studies provide a strong body of evidence to suggest that tea drinking has the capacity to lower the incidence of dental caries.

Although formal proof will be dependent on further large-scale epidemiological studies, extracts of green tea may represent an inexpensive, safe and readily available product with which to combat this very common condition. 


\subsection{Effect of green tea on periodontal disease}

The gingival crevice, a readily formed space between the gums and the tooth margin, typically contains very large numbers of microbes, the majority of which are anaerobes. In periodontal disease, the space enlarges to become a 'pocket', with local inflammation, an increasing number of polymorphs and a serum exudate. The inflamed gum bleeds readily and then recedes; later stages of the disease are characterized by absorption of ligaments and weakening of bone, causing the teeth to loosen. The disease is a major cause of tooth loss in adults. Anaerobic black-pigmented bacteria such as Prevotella spp. and Porphyromonas gingivalis are intimately associated with periodontal disease.

Kaneko et al. (1993) found that a four-week regimen of mouth washing with a dilute catechin solution reduced the mouth odour (halitosis) associated with periodontal disease; it was subsequently established that tea catechins deodorized methyl mercaptan, the main cause of halitosis (Yasuda and Arakawa 1995). These authors showed a clear correlation between efficacy and the antioxidative capacity of individual catechins, with EGCg being more effective than EGC and ECg. In addition, catechin gallates, especially EGCg (active at $250-500 \mu \mathrm{g} / \mathrm{ml}$ ), inhibited growth and adherence to buccal epithelial cells of $P$. gingivalis (Sakanaka et al. 1996). Hirasawa et al. (2002) demonstrated bactericidal activity of green tea catechins at $1 \mathrm{mg} / \mathrm{ml}$ against species of Prevotella and $P$. gingivalis, and found significant reduction in markers of gingivitis after the use of a slow-release buccal delivery system applied over a period of 8 weeks. More recent studies have shown that some virulence factors (toxic end metabolites, protein tyrosine phosphatase and gingipains) associated with these aetiological agents of periodontal disease are neutralized by EGCg (Okamoto et al. 2003, 2004; Sakanaka and Okada 2004).

\subsection{Effects of tea components on the bacterial phenotype}

The major bioactive components of green tea, the catechins, possess only moderate antibacterial activity, although these molecules may, in the foreseeable future, find utility in the treatment of topical and oral infections. Further, it is clear that they are able to aid general wellbeing by, for example, exerting a positive influence on the composition of the gastrointestinal flora, as described earlier. However, unless substantial chemical modifications to the catechin structure can be made that result in significant improvement in both antibacterial efficacy and stability in vivo, these molecules are unlikely to be useful as conventional anti-infective agents for the treatment of severe systemic infections. However, naturally occurring catechins have a wide range of activities on bacteria, not all of which include the capacity to exert bactericidal or bacteriostatic effects, and some of these are showing early promise with regard to modulation of the host - pathogen relationship. New paradigms for the treatment of bacterial infections are badly needed as the utility of conventional antibiotics is being eroded by the spread of multiple drug resistance; catechinmediated effects may serve as useful indicators for the development of novel modalities for the treatment of infection (Taylor et al. 2002). Indirect effects of catechins on bacteria include a capacity to modify antibiotic sensitivity and to alter the expression of factors that determine bacterial virulence.

It was reported by Yam et al. (1998) that extracts of green tea have the capacity to reverse methicillin resistance in MRSA isolates at concentrations much lower than those needed to produce inhibition of bacterial growth. Subsequent examination of a range of individual components of tea has indicated that this activity was attributable to the catechin gallates, with ECg showing greater potency than either EGCg or Cg (Hamilton-Miller and Shah 2000). Japanese groups have also demonstrated that ECg (Shiota et al. 1999) and EGCg (Zhao et al. 2001a) markedly lower the MIC of methicillin, oxacillin and other $\beta$-lactam antibiotics in clinical isolates of MRSA. These findings are significant because $S$. aureus is 
one of the major causes of both nosocomial and community-acquired infections worldwide. The use and overuse of $\beta$-lactam agents, such as methicillin, and other antibiotics has resulted in intense selective pressure on bacterial populations and has led to the emergence of multi-drug resistant bacteria that are threatening our ability to treat serious infections, particularly in hospitals (PHLS Report 2002).

$\beta$-Lactam resistance in $S$. aureus is associated with the presence of a $40-60 \mathrm{~kb}$ chromosomal element (mec), that includes the $m e c A$ gene encoding an additional penicillin binding protein (PBP) termed PBP2a. The PBPs catalyse the insertion and cross-linking of newly synthesized peptidoglycan precursors in to the staphylococcal cell wall; they are the targets for $\beta$-lactam antibiotics (Ehlert 1999). PBP2a has a lower affinity for $\beta$-lactams than other PBPs, in particular its analogue PBP2, and maintains transpeptidase activity in the presence of $\beta$-lactam concentrations that saturate other PBPs (Berger-Bächi and Tschierske 1998). PBP2a usually confers high levels of $\beta$-lactam resistance on strains and is the basis of $\beta$ lactam resistance in the methicillin-resistant genotype of almost all clinical isolates of MRSA. Stapleton et al. (2004a) examined the capacity of a range of catechins and gallates to reverse $\beta$-lactam resistance in 40 clinical isolates of MRSA and found that $12.5-25 \mathrm{mg} / \mathrm{l}$ of ECg reduced MIC values for oxacillin from levels of 256 and $512 \mathrm{mg} / \mathrm{l}$ to $1-4 \mathrm{mg} / \mathrm{l}$. This modification, from full resistance to below the antibiotic breakpoint at which drugs are deemed to be of therapeutic value, raises the possibility that ECg, or stable catechin gallate analogues, could be used in combination with suitable $\beta$-lactam agents to treat MRSA infections in the clinic. This study also determined that the gallate moiety of ECg was essential for oxacillin-modulating activity, although gallic acid did not increase the sensitivity of MRSA to $\beta$-lactam agents. In further studies of the relationship between structure and $\beta$-lactam-modifying function, these researchers determined that modifications to the B-ring (Figure 1) of ECg affected the degree of resistance modulation; in addition to functional differences between $\mathrm{ECg}$ and $\mathrm{EGCg}$, which differ only in the degree of hydroxylation of the B-ring, unnatural mono- and dihydroxy analogues of $\mathrm{ECg}$ were able to fully sensitize MRSA strains to oxacillin (Anderson et al. 2005b).

Recent data from in vivo experiments support the notion that green tea components may display synergy with conventional antibiotics against Gram-negative bacteria. Isogai et al. (2001) determined that gnotobiotic mice fed extracts of $C$. sinensis leaves were protected against oral challenge with enterohaemorrhagic Escherichia coli $\mathrm{O} 157$ when given in combination with levofloxacin. Although complete elimination of the bacteria from the animals was not achieved, the combination increased survival rate and reduced organ damage when compared to mice fed a conventional diet or given levofloxacin alone. These observations might be related to catechin-mediated effects on the bacterial phenotype, to inhibition of inflammation or ulceration of the gastrointestinal mucosa or to enhancement of macrophage function, as recently noted for EGCg (Yamamoto et al. 2004). Similarly, the catechin fraction of green tea acted synergistically with ciprofloxacin in a chronic bacterial prostatitis model in the rat (Lee et al. 2005).

Horizontal spread of plasmid-borne antibiotic resistance genes has contributed greatly to the present day incidence of multiple drug resistance; the observation by Zhao et al. (2001b), that EGCg diminishes the transfer of conjugative drug resistance plasmids in a dosedependent manner, deserves attention. As a significant level of plasmid-mediated gene transfer is likely to take place in the gastrointestinal tract (the primary habitat of many problematical multi-resistant opportunistic pathogens), the consumption of green tea may be beneficial in reducing or even preventing resistance plasmid transfer in enteric bacteria. The wide-ranging effects that catechin gallates have on the modulation of bacterial drug resistance has recently been emphasized by the novel observation that sub-inhibitory concentrations of EGCg are able to reverse tetracycline resistance in staphylococci by 
inhibition of the Tet(K) efflux pump, in addition to further sensitizing susceptible staphylococcal isolates to this antibiotic (Roccaro et al. 2004). Despite this plethora of observations on the multifaceted nature of the antibacterial effects of catechins, there is little published data on the mechanisms that underpin this work. Any attempt to develop hypotheses that account for these effects must take into account the potential of catechin gallates to modify the secretion of key proteins in both Gram-positive and Gram-negative bacteria. For example, these molecules are known to interfere with the expression of $\beta$ lactamases in staphylococci (Yam et al. 1998, Zhao et al. 2002) and to inhibit the extracellular release of verotoxin from enterohaemmorrhagic E. coli O157 (Sugita-Konishi et al. 1999). The current understanding of the interactions that might account for these activities against bacteria is reviewed below.

\subsection{Interactions of catechins with bacteria}

The literature is replete with reports of antibacterial activity associated with extracts from an enormous range of plants. For example, Brantner and Grein (1994) produced a large number of aqueous extracts of members of 28 different plant families and found that the majority exhibited some level of antibacterial activity. In virtually all cases, however, inhibition of bacterial growth was extremely weak and it is difficult to envisage that this information could be put to practical use. In a similar fashion, the main bioactive components of green tea have only modest direct activity and will not find utility as conventional antibacterial agents other than in oral or topical infections. Thus, studies aimed at determining the mechanism of antibacterial action of catechins are hampered by the high concentrations that must be used to elicit marginal effects. In contrast, the capacity of catechins to modify the bacterial phenotype occurs at concentrations that may be achievable in vivo and these effects are likely to involve more precise and selective interactions.

In attempting to explain the weak antibacterial action of EGCg, Ikigai et al. (1993) concluded that high EGCg concentrations irreversibly damaged the bacterial cytoplasmic membrane, on the basis that phosphatidyl choline (PC) liposomes leaked fluorescent dye following exposure to the compound. However, the introduction of charged lipids into the liposomal bilayer, reflecting more appropriately the composition of the cytoplasmic membranes of Gram-positive and Gram-negative bacteria, reduced the extent of this damage. It has recently been claimed that cytoplasmic membrane damage results from the generation of hydrogen peroxide by EGCg within the bilayer (Arakawa et al. 2004). It has also been suggested that the antibacterial action of EGCg against Stenotrophomonas maltophila, a Gram-negative opportunistic pathogen of growing importance, is due to its capacity to inhibit the cytoplasmic enzyme dihydrofolate reductase (DHFR; NavarroMartínez et al. 2005). The catechin was shown to inhibit DHFR purified from both trimethoprim-susceptible and trimethoprim-resistant $S$. maltophila clinical isolates. Other workers have examined the capacity of EGCg to inhibit the type II fatty acid synthesis system located in the cytoplasmic compartment of E. coli and other bacteria. Zhang and Rock (2004) noted that EGCg potently inhibited the FabG and FabI reductase steps in the fatty acid elongation cycle and concluded that the capacity of EGCg to interfere with these $\mathrm{NAD}(\mathrm{P})$-dependent intracellular processes must be taken into account when assessing the mechanism of antibacterial action of plant polyphenols. Unfortunately, evidence has accumulated to show that EGCg and other catechins do not gain entry into cells but exert their effects from the cell membrane; these data will be reviewed below.

Growth of $S$. aureus, including MRSA, in the presence of sub-inhibitory concentrations of catechin gallates has a profound effect on the architecture of the cell wall. Aqueous extracts of green tea (Hamilton-Miller and Shah 1999) or ECg (Stapleton et al. 2005) have been shown to increase cell wall thickness and to stimulate the formation of pseudo-multicellular aggregates. Cell wall autolysins are involved in both cell wall turnover and cell separation 
and we have therefore investigated the effect of ECg on autolysin production and activity (Stapleton et al. 2005). Quantities of autolysins released into the culture medium were significantly reduced when staphylococci were grown in the presence of ECg; increased amounts were found in the thickened cell wall, indicating that growth in ECg compromised the processing or in situ activity of the autolysins. Furthermore, ECg stimulated the release of lipoteichoic acid from the cell wall (Stapleton et al. 2005); this polymer, anchored into the staphyloccocal cytoplasmic membrane, is known to modulate the sensitivity of MRSA to $\beta$ lactam antibiotics (Neuhaus and Baddiley 2003) and appears to be displaced by ECgmediated alterations to the physical structure of the bilayer. The phenotype generated by growth of MRSA strains in ECg is strikingly similar to that noted for vancomycinintermediate resistant $S$. aureus clinical isolates, so-called VISA strains. These also produce abnormally thick cell walls, separate poorly when grown in antibiotic-free medium and show decreased cross-linkage of the peptidoglycan and altered teichoic acid content compared to vancomycin-susceptible progenitors (Sieradski and Tomasz 2003). Interestingly, VISA isolates also have an increased susceptibility to $\beta$-lactam agents. The ECg-induced changes that we have observed may go some way towards accounting for the increased susceptibility of MRSA strains to $\beta$-lactam antibiotics induced by ECg at subinhibitory concentrations, and could result from perturbation of the cytoplasmic membrane by ECg.

Recent work has shown that catechins in the nanomole range are able to modulate the structure and function of model membranes, due to their capacity to partition into the phospholipid palisade (Hashimoto et al. 1999; Kumazawa et al. 2004). Catechin gallates bound more avidly than either EC or EGC to small unilamellar vesicles produced from PC, and ECg had a greater affinity for the bilayer than EGCg (Hashimoto et al. 1999). The relative affinity of catechins for membranes reflected their partition coefficients in $n$-octanol saline (Hashimoto et al. 1999) and their capacity to modulate $\beta$-lactam resistance in MRSA strains (Stapleton et al. 2004a). Catechins with epi (cis) stereochemistry partitioned into PC liposomes more readily than their non-epi (trans) configured counterparts (Kajiya et al. 2001); in epicatechins such as ECg and EGCg, the hydrophobic domain in the region of the ester bond and C-ring (Figure 2) is relatively exposed and therefore liable to perturb the bilayer.

These observations suggest that membrane interactions of catechins are governed by the degree of hydroxylation of the B-ring, the presence of a gallate moiety and the stereochemistry of the C-ring. Catechin gallates, especially ECg, penetrate into the hydrophobic core of the membrane, whereas EC and EGC adopt a more superficial location close to the phospholipid-water interface (Caturla et al. 2003). This can be rationalized by looking at space filling models of typical stable conformations for $\mathrm{ECg}$ and $\mathrm{Cg}$ (Figure 2). The hydrophobic domain of $\mathrm{Cg}$ is encumbered by neighbouring hydrophilic domains, due to the trans stereochemistry of the structure. Galloyl amide (compound (1) in Figure 2), which we have recently synthesized, is fully active as an oxacillin resistance modulator (Anderson et al. 2005b) and offers a nearly identical hydrophobic domain to ECg, as it possesses the EC cis stereochemistry. Note that removal of one hydroxyl group from ECg, giving compound (2) as shown in Figure 2, further extends the dimensions of the hydrophilic domain; compound (2) was found to possess high-level $\beta$-lactam-modulating activity in MRSA (Anderson et al. 2005a). ECg affects the physical properties of PC and phosphatidyl ethanolamine bilayers, inducing tight packing of the phospholipid acyl chains (Caturla et al. 2003). Such changes in the ordered structure of the bilayer are influenced by salt concentration (Kajiya et al. 2002), pH (Kajiya et al. 2002), the nature of the phospholipid (Kajiya et al. 2002; Caturla et al. 2003) and the presence of other catechins (Hashimoto et al. 1999), and are likely to affect transport of materials across the membrane. 
These observations suggest that ECg and other catechin gallates sensitize MRSA strains to $\beta$-lactam antibiotics by virtue of their capacity to bind to and penetrate the cytoplasmic membrane. Their intercalation deep into the hydrocarbon core of the bilayer is enhanced by non-galloyl catechins that bind superficially to the outer surface of the membrane and create conditions favourable for penetration. This membrane perturbation increases the lipid order, producing tightly packed and extended acyl chains in the bilayer; it also reduces the efficiency of function of a number of membrane proteins such as the PBPs, resulting in changes to the architecture of the cell wall. Catechin-induced changes may also affect the traffic of proteins across the membrane. Penetration of catechin gallates into the bilayer displaces lipoteichoic acid, a macromolecule known to modulate the sensitivity of MRSA to $\beta$-lactam antibiotics. We are currently subjecting this model to rigorous investigation.

\section{Pharmaceutical considerations}

There are a small number of reports in the literature demonstrating that under certain conditions catechins, in particular catechin gallates, are unstable. EGCg degrades in neutral solutions ( $\mathrm{pH}$ 6.5-7.8) to yield several products, including the dimeric compounds theasinensin A and theasinensin D (Hatano et al. 2003). This study was performed in the context of an investigation of the reversal of oxacillin resistance in MRSA and it was noted that theasinensin A was a potent modifier of resistance to this drug. However, it remains to be determined if the data presented by various groups on the $\beta$-lactam-modifying capacity of EGCg and ECg are in part due to degradation products of these tea constituents. Catechins are known to be more stable in an acidic environment (Pomponio et al. 2003) but very little information is available in the literature of their stability in biological milieu; this is an area that warrants urgent investigation.

Following oral administration, naturally occurring catechin gallates are rapidly metabolized to inactive products by intestinal bacteria (Kohri et al. 2001), due to the presence of an esterase susceptible bond linking the B-ring to the A-C fused ring system (Figure 1). After removal of the galloyl group from EGCg, EGC undergoes reduction, cleavage, dehydroxylation, further decomposition and lactonization to form hydroxyphenyl- $\gamma$ valerolactone products that are excreted via urine and faeces (Kohri et al. 2001). Replacing the ester bond with an amide linkage group stabilizes the molecule by preventing the initial cleavage step (Anderson et al. 2005b). In man, systemic availability of EGCg increases with the oral dose and significant levels were detected in plasma (Chow et al. 2001). The poor oral bioavailability in man of catechin gallates is enhanced by administration after overnight fasting, which is likely to increase the biological response to the agent (Chow et al. 2005). These fundamental observations of catechin metabolism should inform on strategies for the development of dosage forms for use in man. It remains to be seen whether systemic administration of EGCg or ECg achieves a greater bioavailability than oral dosing.

\section{Acknowledgments}

We thank the Medical Research Council for financial support through a Strategic Grant Award.

\section{Biography}

Peter Taylor is Professor of Microbiology at the London School of Pharmacy and has collaborated with Professor Hamilton-Miller for a number of years. His interests are in identifying and developing new modalities for the treatment of infection, in particular those that suppress or abrogate the emergence of the drug resistant genotype. Jeremy HamiltonMiller is Emeritus Professor of Medical Microbiology at the Royal Free and University College Medical School. He has a broad range of interests in the area of antibacterial 
chemotherapy and has a particular fascination with the use of natural products and probiotics in the treatment and control of infection. Paul Stapleton is Senior Research and Teaching Fellow at the London School of Pharmacy and has a particular interest in the molecular biology and clinical epidemiology of the staphylococcus.

\section{Abbreviations}

$\begin{array}{ll}\text { MRSA } & \text { methicillin-resistant Staphylococcus aureus } \\ \text { MIC } & \text { minimum inhibitory concentration } \\ \text { HIV } & \text { human immunodeficiency virus } \\ \text { PBP } & \text { penicillin binding protein } \\ \text { DHFR } & \text { dihydrofolate reductase } \\ \text { PC } & \text { phosphatidyl choline } \\ \text { NAD(P) } & \text { nicotinamide adenine dinucleotide (phosphate) } \\ \text { EGCg } & \text { (-)-epigallocatechin gallate } \\ \text { ECg } & \text { (-)-epicatechin gallate } \\ \text { Cg } & \text { (-)-catechin gallate }\end{array}$

\section{References}

Agarwal R, Katiyar SK, Zaidi SI, Mukhtar H. Inhibition of skin tumor promoter-caused induction of epidermal ornithine decarboxylase in SENCAR mice by polyphenolic fraction isolated from green tea and its individual epicatechin derivatives. Cancer Research. 1992; 52:3582-3588. [PubMed: 1617628]

Anderson JC, Headley C, Stapleton PD, Taylor PW. Asymmetric total synthesis of B-ring modified (-)-epicatechin gallate analogues and their modulation of $\beta$-lactam resistance in Staphylococcus aureus. Tetrahedron. 2005a; 61:7703-7711. [PubMed: 19330042]

Anderson JC, Headley C, Stapleton PD, Taylor PW. Synthesis and antibacterial activity of a hydrolytically stable (-)-epicatechin gallate analogue for the modulation of $\beta$-lactam resistance in Staphylococcus aureus. Bioorganic and Medicinal Chemistry Letters. 2005b; 15:2633-2635. [PubMed: 15863332]

Arakawa H, Maeda M, Okubo S, Shimamura T. Role of hydrogen peroxide in bactericidal action of catechin. Biological and Pharmaceutical Bulletin. 2004; 27:277-281. [PubMed: 14993788]

Bell SJ, Goodrick GK. A functional food product for the management of weight. Critical Reviews in Food Science and Nutrition. 2002; 42:163-178. [PubMed: 11934132]

Berger-Bächi B, Tschierske M. Role of Fem factors in methicillin resistance. Drug Resistance Updates. 1998; 1:325-335. [PubMed: 17092813]

Brantner A, Grein E. Antibacterial activity of plant extracts used externally in traditional medicine. Journal of Ethnopharmacology. 1994; 44:35-40. [PubMed: 7990502]

Caturla N, Vera-Semper E, Villalaín J, Reyes Mateo C, Micol V. The relationship between the antioxidant and the antibacterial properties of galloylated catechins and the structure of phospholipid model membranes. Free Radical Biology and Medicine. 2003; 34:648-662. [PubMed: 12633742]

Chen D, Daniel KG, Kuhn DJ, Kazi A, Bhuiyan M, Li L, Wang Z, Wan SB, Lam WH, Chan TH, Dou QP. Green tea and tea polyphenols in cancer prevention. Frontiers in Bioscience. 2004; 9:26182631. [PubMed: 15358585]

Chosa H, Toda M, Okubo S, Hara Y, Shimamura T. Antimicrobial and microbicidal activities of tea and catechins against Mycoplasma. Journal of the Japanese Association for Infectious Diseases. 1992; 66:606-611. [PubMed: 1402093] 
Chow H-HS, Cai Y, Alberts DS, Hakin I, Dorr R, Shahi F, Crowell JA, Yang CS, Hara Y. Phase I pharmacokinetic study of tea polyphenols following single-dose administration of epigallocatechin gallate and Polyphenon E. Cancer Epidemiology, Biomarkers and Prevention. 2001; 10:53-58. [PubMed: 11205489]

Chow H-HS, Cai Y, Hakim IA, Crowell JA, Shahi F, Brooks CA, Dorr RT, Hara Y, Alberts DS. Pharmacokinetics and safety of green tea polyphenols after multiple-dose administration of epigallocatechin gallate and polyphenon $\mathrm{E}$ in healthy individuals. Clinical Cancer Research. 2003; 9:3312-3319. [PubMed: 12960117]

Chow H-HS, Hakim IA, Vining DR, Crowell JA, Ranger-Moore J, Chew WM, Celaya CA, Rodney SR, Hara Y, Alberts DS. Effects of dosing condition on the oral bioavailability of green tea catechins after single-dose administration of Polyphenon E in healthy individuals. Clinical Cancer Research. 2005; 11:4627-4633. [PubMed: 15958649]

Chung JY, Huang C, Meng X, Dong Z, Yang CS. Inhibition of activator protein 1 activity and cell growth by purified green tea and black tea polyphenols in H-ras-transformed cells: structureactivity relationship and mechanisms involved. Cancer Research. 1999; 59:4610-4617. [PubMed: 10493515]

Devulapalle KS, Mooser G. Glucosyltransferase inactivation reduces dental caries. Journal of Dental Research. 2001; 80:466-469. [PubMed: 11332534]

Downer MC, Drugan CS, Blinkhorn AS. Dental caries experience of British children in an international context. Community Dental Health. 2005; 22:86-93. [PubMed: 15984133]

Ehlert K. Methicillin-resistance in Staphylococcus aureus - molecular basis, novel targets and antibiotic therapy. Current Pharmaceutical Design. 1999; 5:45-55. [PubMed: 10066883]

Elvin-Lewis M, Steelman R. The anticariogenic effects of tea drinking among Dallas school children. Journal of Dental Research. 1986; 65:198.

Fassina G, Buffa A, Benelli R, Varnier OE, Noonan DM, Albini A. Polyphenolic antioxidant (-)epigallocatechin-3-gallate from green tea as a candidate anti-HIV agent. AIDS. 2002; 16:939-941. [PubMed: 11919502]

Goto K, Kanaya S, Nishikawa T. The influence of tea catechins on faecal flora of elderly residents in long-term care facilities. Annals of Long-Term Care: Clinical Care and Aging. 1998; 6:43-48.

Graham H. Green tea composition, consumption and polyphenol chemistry. Preventive Medicine. 1992; 21:334-350. [PubMed: 1614995]

Hamilton-Miller, JMT. Microbial properties of tea infusions. In: Schubert, R.; Spiro, M., editors. Chemical and biological properties of tea infusions. Frankfurt, Germany: U \& M; 1997. p. 63-74.

Hamilton-Miller JMT. Anti-cariogenic effects of tea (Camellia sinensis). Journal of Medical Microbiology. 2001; 50:299-302. [PubMed: 11289514]

Hamilton-Miller JMT, Shah S. Disorganisation of cell division of methicillin-resistant Staphylococcus aureus by a component of tea (Camellia sinensis): a study by electron microscopy. FEMS Microbiology Letters. 1999; 176:463-469. [PubMed: 10427729]

Hamilton-Miller JMT, Shah S. Activity of the tea component epicatechin gallate and analogues against methicillin-resistant Staphylococcus aureus. Journal of Antimicrobial Chemotherapy. 2000; 46:852-853. [PubMed: 11062217]

Hara, Y. Green tea: health benefits and applications. New York, USA: Marcel Dekker; 2001.

Hara, Y.; Hattori, M. Method of preventing the formation or aggrevation of dental plaque and method for reducing cariogenesis. United States Patent. 5204089. 1992.

Hara Y, Honda M. The inhibition of a-amylase by tea polyphenols. Agricultural and Biological Chemistry. 1990; 54:1939-194.

Hashimoto T, Kumazawa S, Nanjo F, Hara Y, Nakayama T. Interaction of tea catechins with lipid bilayers investigated with liposome systems. Bioscience, Biotechnology and Biochemistry. 1999; 63:2252-2255. [PubMed: 10664864]

Hatano T, Kusuda M, Hori M, Shiota S, Tsuchiya T, Yoshida T. Theasinensin A, a tea polyphenol formed from (-)-epigallocatechin gallate, suppresses antibiotic resistance of methicillin-resistant Staphylococcus aureus. Planta Medica. 2003; 69:984-989. [PubMed: 14735433] 
Hattori M, Kusumoto IT, Namba T, Ishigami T, Hara Y. Effect of tea polyphenols on glucan synthesis by glucosyltransferase from Streptococcus mutans. Chemical and Pharmaceutical Bulletin. 1990; 38:717-720. [PubMed: 2140716]

Hertog M, Feskens E, Hollman P, Katan M, Kromhout D. Dietary antioxidant flavonoids and risk of coronary heart disease. The Zutphen Elderly Study. The Lancet. 1993; 342:1007-1011. [PubMed: 8105262]

Hirasawa M, Takada K, Makimura M, Otake S. Improvement of periodontal status by green tea catechins using a local delivery system: a clinical pilot study. Journal of Periodontal Research. 2002; 37:433-438. [PubMed: 12472837]

Hirasawa M, Takada K. Multiple effects of green tea catechin on the natifungal activity of antimycotics against Candida albicans. Journal of Antimicrobial Chemotherapy. 2004; 53:225229. [PubMed: 14688042]

Hsu S. Green tea and the skin. Journal of the American Academy of Dermatology. 2005; 52:10491059. [PubMed: 15928624]

Ikigai H, Nakae T, Hara Y, Shimamura T. Bactericidal catechins damage the lipid bilayer. Biochimica et Biophysica Acta. 1993; 1147:132-136. [PubMed: 8466924]

Isogai E, Isogai H, Hirose K, Hayashi S, Oguma K. In vivo synergy between green tea extract and levofloxacin against enterohemorrhagic Escherichia coli O157 infection. Current Microbiology. 2001; 42:248-251. [PubMed: 11178724]

Joiner A, Muller D, Elofsson UM, Arnebrant T. Ellipsometry analysis of the in vitro adsorption of tea polyphenols onto salivary pellicles. European Journal of Oral Science. 2004; 112:510-515. [PubMed: 15560834]

Jones C, Woods K, Whittle G, Worthington H, Taylor G. Sugar, drinks, deprivation and dental caries in 14-year old children in the north west of England. Community Dental Health. 1999; 16:68-71. [PubMed: 10641059]

Kajiya K, Kumazawa S, Nakayama T. Steric effects on interaction of tea catechins with lipid bilayers. Bioscience, Biotechnology and Biochemistry. 2001; 65:2638-2643. [PubMed: 11826958]

Kajiya K, Kumazawa S, Nakayama T. Effects of external factors on the interaction of tea catechins with lipid bilayers. Bioscience, Biotechnology and Biochemistry. 2002; 66:2330-2335. [PubMed: 12506968]

Kaneko K, Shimano N, Suzuki Y, Nakamukai M, Ikazaki R, Ishida N, Kanayasu E, Karuda T, Takihara T, Sakane I, Yayabe F, Matsui T. Effects of tea catechins on oral odor and dental plaque. Oral Therapeutics and Pharmacology. 1993; 12:189-197.

Kashket S, Paolino VJ. Inhibition of salivary amylase by water-soluble extracts of tea. Archives of Oral Biology. 1988; 33:845-846. [PubMed: 2476976]

Kashket S, Paolino VJ, Lewis DA, van Houte J. In vitro inhibition of glucosyltransferase from the dental plaque bacterium Streptococcus mutans by common beverages and food extracts. Archives of Oral Biology. 1985; 30:821-826. [PubMed: 2938561]

Keli S, Hertog M, Feskens E, Kromhout D. Flavonoids, antioxidant vitamins and risk of stroke. Archives of Internal Medicine. 1995; 154:637-642.

Kohri T, Matsumoto N, Yamakawa M, Suzuki M, Nanjo F, Hara Y, Oku N. Metabolic fate of (-)$\left[4-{ }^{3} \mathrm{H}\right]$ epigallocatechin gallate in rats after oral administration. Journal of Agricultural and Food Chemistry. 2001; 49:4102-4112. [PubMed: 11513717]

Kawamura J, Takeo T. Antibacterial activity of tea catechin to Streptococcus mutans. Journal of the Japanese Society of Food Science and Technology. 1989; 36:463-467.

Kubo I, Muroi H, Himejima M. Antimicrobial activity of green tea flavor components and their combination effects. Journal of Agricultural and Food Chemistry. 1992; 40:245-248.

Kumazawa S, Kajiya K, Naito A, Saito H, Tuzi S, Tanio M, Suzuki M, Nanjo F, Suzuki E, Nakayama T. Direct evidence of interaction of a green tea polyphenol, epigallocatechin gallate, with lipid bilayers by solid-state nuclear magnetic resonance. Bioscience, Biotechnology Biochemistry. 2004; 68:1743-1747. [PubMed: 15322359]

Laughton MJ, Evans PJ, Moroney MA, Hoult JR, Halliwell B. Inhibition of mammalian 5lipoxygenase and cyclo-oxygenase by flavonoids and phenolic dietary additives. Relationship to

Food Sci Technol Bull. Author manuscript; available in PMC 2009 October 19. 
antioxidant activity and to iron ion-reducing ability. Biochemical Pharmacology. 1991; 42:16731681. [PubMed: 1656994]

Lee YS, Han CH, Kang SH, Lee S-J, Kim SW, Shin OR, Sim Y-C, Lee S-J, Cho Y-H. Synergistic effect between catechin and ciprofloxacin on chronic bacterial prostatitis rat model. International Journal of Urology. 2005; 12:383-389. [PubMed: 15948727]

Liu T, Chi Y. Experimental study on polyphenol anti-plaque effect in humans. Chinese Journal of Stomatology. 2000; 35:383-384. [PubMed: 11780252]

McKay DL, Blumberg JB. The role of tea in human health: an update. Journal of the American College of Nutrition. 2002; 21:1-13. [PubMed: 11838881]

McNaught JG. On the action of cold or lukewarm tea on Bacillus typhosus. Journal of the Royal Army Medical Corps. 1906; 7:372-373.

Navarro-Martínez MD, Navarro-Perán E, Cabezas-Herrera J, Ruiz-Gómez J, Garcáa-Cánovas F, Rodríguez-López JN. Antifolate activity of epigallocatechin against Stenotrophomonas maltophilia. Antimicrobial Agents and Chemotherapy. 2005; 49:2914-2920. [PubMed: 15980368]

Neuhaus FC, Baddiley J. A continuum of anionic charge: structures and functions of D-alanyl-teichoic acids in Gram-positive bacteria. Microbiology and Molecular Biology Reviews. 2003; 67:686723. [PubMed: 14665680]

Okamoto M, Leung K-P, Ansai T, Sugimoto A, Maeda N. Inhibitory effects of green tea catechins on protein tyrosine phosphatase in Prevotella intermedia. Oral Microbiology and Immunology. 2003; 18:192-195. [PubMed: 12753472]

Okamoto M, Sugimoto A, Leuing K-P, Nakayama K, Kamaguchi A, Maeda N. Inhibitory effect of green tea catechins on cysteine proteinases in Porphyromonas gingivalis. Oral Microbiology and Immunology. 2004; 19:118-120. [PubMed: 14871352]

Okubo S, Toda M, Hara Y, Shimamura T. Antifungal and antifungicidal activities of tea extract and catechin against Trichophyton. Japanese Journal of Bacteriology. 1991; 46:509-514. [PubMed: 2062004]

Onisi M. The feasibility of a tea drinking program for dental public health in primary schools. Journal of Dental Health. 1985; 35:401-412.

Onisi M, Shimura N, Nakamura C, Sato M. A field test on the caries preventive effect of tea drinking. Journal of Dental Health. 1981; 31:13-19.

Otake S, Makimura M, Kuroki T, Nishihara Y, Hirasawa M. Anticaries effects of polyphenolic compounds from Japanese green tea. Caries Research. 1991; 25:438-443. [PubMed: 1667297]

Paveto C, Güida MC, Estava MI, Martino V, Coussio J, Flawiá MM, Torres HN. Anti-Typanosoma cruzi activity of green tea (Camellia sinensis) catechins. Antimicrobial Agents and Chemotherapy. 2004; 48:69-74. [PubMed: 14693520]

PHLS Report. Antimicrobial Resistance in 2000: England and Wales. London, UK: Public Health Laboratory Service; 2002.

Pomponio R, Gotti R, Luppi B, Cavrini V. Microemulsion electrokinetic chromatography for the analysis of green tea catechins: effect of the cosurfactant on the separation selectivity. Electrophoresis. 2003; 24:1658-1667. [PubMed: 12761797]

Rasheed A, Haider M. Antibacterial activity of Camellia sinensis extracts against dental caries. Archives of Pharmaceutical Research. 1998; 21:348-352. [PubMed: 9875456]

Roccaro AS, Blanco AR, Giuliano F, Rusciano D, Enea V. Epigallocatechin gallate enhances the activity in staphylococci by inhibiting its efflux from bacterial cells. Antimicrobial Agents and Chemotherapy. 2004; 48:1968-1973. [PubMed: 15155186]

Sakanaka S, Kim M, Taniguchi M, Yamamoto T. Antibacterial substances in Japanese green tea extract against Streptococcus mutans, a cariogenic bacterium. Agricultural and Biological Chemistry. 1989; 53:2307-2311.

Sakanaka S, Aizawa M, Kim M, Yamamoto T. Inhibitory effects of green tea polyphenols on growth and cellular adherence of an oral bacterium, Porphyromonas gingivalis. Bioscience, Biotechnology and Biochemistry. 1996; 60:745-749. [PubMed: 8704303]

Sakanaka S, Okada Y. Inhibitory effects of green tea polyphenols on the production of a virulence factor of the periodontal-disease-causing anaerobic bacterium Porphyromonas gingivalis. Journal of Agricultural and Food Chemistry. 2004; 52:1688-1692. [PubMed: 15030231] 
Scott BC, Butler J, Halliwell B, Aruoma OI. Evaluation of the antioxidant actions of ferulic acid and catechins. Free Radical Research Communications. 1993; 19:241-253. [PubMed: 7507456]

Sharquie KE, al-Turfi IA, al-Salloum SM. The antibacterial activity of tea in vitro and in vivo in patients with impetigo contagiosa. Journal of Dermatology. 2000; 27:706-710. [PubMed: 11138536]

Shiota S, Shimizu M, Mizushima T, Ito H, Hatano T, Yoshida T, Tsuchiya T. Marked reduction in the minimum inhibitory concentration (MIC) of $\beta$-lactams in methicillin-resistant Staphylococcus aureus produced by epicatechin gallate, an ingredient of green tea (Camellia sinensis). Biological and Pharmaceutical Bulletin. 1999; 22:1388-1390. [PubMed: 10746177]

Sieradski K, Tomasz A. Alterations of cell wall structure and metabolism accompany reduced susceptibility to vancomycin in an isogenic series of clinical isolates of Staphylococcus aureus. Journal of Bacteriology. 2003; 185:7103-7110. [PubMed: 14645269]

Song J-M, Lee K-H, Seong B-L. Antiviral effect of catechins in green tea on influenza virus. Antiviral Research. 2005 E-publication $29^{\text {th }}$ August.

Spencer JP, Schroeter H, Kuhnle G, Srai SK, Tyrrell RM, Hahn U, Rice-Evans C. Epicatechin and its in vivo metabolite, $3^{\prime}$-O-methyl epicatechin, protect human fibroblasts from oxidative stressinduced cell death involving caspase-3 activation. Biochemical Journal. 2001; 354:493-500. [PubMed: 11237853]

Stapleton PD, Shah S, Anderson JC, Hara Y, Hamilton-Miller JMT, Taylor PW. Modulation of $\beta$ lactam resistance in Staphylococcus aureus by catechins and gallates. International Journal of Antimicrobial Agents. 2004a; 23:462-467. [PubMed: 15120724]

Stapleton PD, Shah S, Hamilton-Miller JMT, Hara Y, Nagaoka Y, Kumagai A, Uesato S, Taylor PW. Anti-Staphylococcus aureus activity and oxacillin resistance modulating capacity of 3- $O$-acylcatechins. International Journal of Antimicrobial Agents. 2004b; 24:374-380. [PubMed: 15380264]

Stapleton, PD.; Shah, S.; Taylor, PW. Altered cell surface properties and decreased autolytic activity in methicillin-resistant Staphylococcus aureus by epicatechin gallate; International Union of Microbiological Societies; San Francisco, USA: 2005. Abstract B-852

Sugita-Konishi Y, Hara-Kubo Y, Amano F, Okubo T, Aoi N, Iwaki M, Kumagai S. Epigallocatechin gallate and gallocatechin gallate in green tea catechins inhibit extracellular release of Vero toxin from enterohemorrhagic Escherichia coli O157:H7. Biochimica et Biophysica Acta. 1999; 1472:42-50. [PubMed: 10572924]

Taylor PW, Stapleton PD, Luzio JP. New ways to treat bacterial infections. Drug Discovery Today. 2002; 7:1086-1091. [PubMed: 12546840]

Williamson G, Manach C. Bioavailability and bioefficacy of polyphenols in humans. II. Review of 93 intervention studies. American Journal of Clinical Nutrition. 2005; 81(Suppl.):243S-255S. [PubMed: 15640487]

Xiao Y, Liu T, Zhan L, Zhou X. The effects of tea polyphenols on the adherence of cariogenic bacterium to the salivary acquired pellicle in vitro. West China Journal of Stomatology. 2000; 18:336-339. [PubMed: 12539656]

Yam TS, Shah S, Hamilton-Miller JMT. Microbiological activity of whole and fractionated crude extracts of tea (Camellia sinensis), and of tea components. FEMS Microbiology Letters. 1997; 152:169-174. [PubMed: 9228784]

Yam TS, Hamilton-Miller JMT, Shah S. The effect of a component of tea (Camellia sinensis) on methicillin resistance, PBP2' synthesis, and $\beta$-lactamase production in Staphylococcus aureus. Journal of Antimicrobial Chemotherapy. 1998; 42:211-216. [PubMed: 9738838]

Yamamoto Y, Matsunaga K, Friedman H. Protective effects of green tea catechins on alveolar macrophages against bacterial infections. Biofactors. 2004; 21:119-121. [PubMed: 15630181]

Yasuda H, Arakawa T. Deodorizing mechanism of (-)-epigallocatechin against methyl mercaptan. Bioscience, Biotechnology and Biochemistry. 1995; 59:1232-1236.

You SQ. Study on feasibility of Chinese green tea polyphenols for preventing dental caries. Chinese Journal of Stomatology. 1993; 28:197-199. [PubMed: 8174399]

Zhang J, Kashket S. Inhibition of salivary amylase by black and green teas and their effects on the intraoral hydrolysis of starch. Caries Research. 1998; 32:233-238. [PubMed: 9577990] 
Zhang Y-M, Rock CO. Evaluation of epigallocatechin gallate and related plant polyphenols as inhibitors of the FabG and FabI reductases of bacterial type II fatty-acid synthase. Journal of Biological Chemistry. 2004; 279:30994-31001. [PubMed: 15133034]

Zhao W-H, Hu Z-Q, Okubo S, Hara Y, Shimamura T. Mechanism of synergy between epigallocatechin gallate and $\beta$-lactams against methicillin-resistant Staphylococcus aureus. Antimicrobial Agents and Chemotherapy. 2001a; 45:1737-1742. [PubMed: 11353619]

Zhao W-H, Hu Z-Q, Hara Y, Shimamura T. Inhibition by epigallocatechin gallate (EGCg) of conjugative R plasmid transfer in Escherichia coli. Journal of Infection and Chemotherapy. 2001b; 7:195-197. [PubMed: 11810584]

Zhao W-H, Hu Z-Q, Hara Y, Shimamura T. Inhibition of penicillinase by epigallocatechin gallate resulting in resoration of antibacterial activity of penicillin against penicillinase-producing Staphylococcus aureus. Antimicrobial Agents and Chemotherapy. 2002; 46:2266-2268. [PubMed: 12069986] 

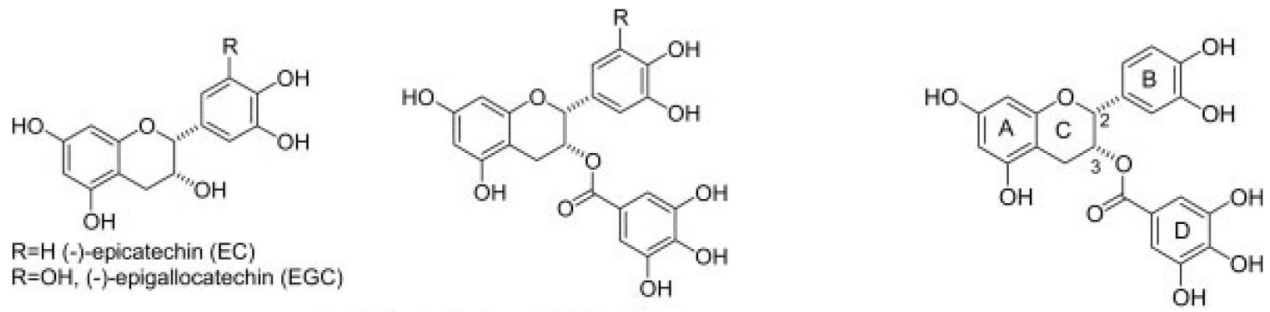

$\mathrm{R}=\mathrm{H}(-)$-epicatechin gallate $(\mathrm{ECg})$

$\mathrm{R}=\mathrm{OH},(-)$-epigallocatechin gallate $(\mathrm{EGCg})$

Figure 1.

Structure of catechins. Ring nomenclature assignment is shown for $\mathrm{ECg}$. 

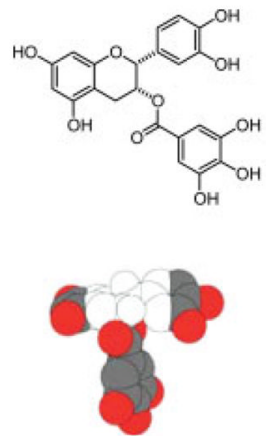

$\mathrm{ECg}$
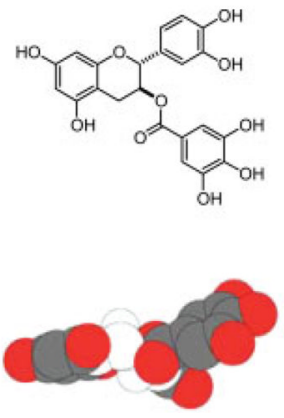

$\mathrm{Cg}$
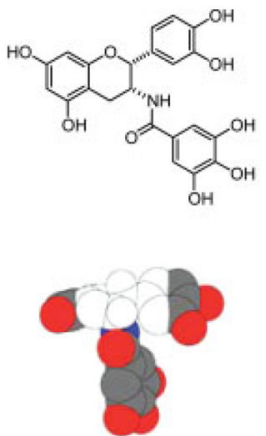

(1)
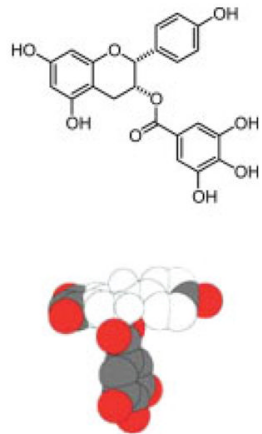

(2)

Figure 2.

Space filling models of natural and synthetically modified catechins. (1) galloyl amide derivative of ECg; (2) monohydroxylated B-ring derivative of ECg. The colourless region is the hydrophobic domain and the dark coloured region the hydrophilic domain. Courtesy of Professor J.C. Anderson, University of Nottingham, UK. 